\title{
Report on the Fifth Congress of the Asian Society for Neuroanesthesia and Critical Care held in Singapore
}

\author{
Chong C. Ted ${ }^{1} \quad$ See J. Jian ${ }^{1} \quad$ Girija P. Rath ${ }^{2} \quad$ Kwek T. Kiat ${ }^{1}$
}

1Department of Anaesthesiology, Intensive Care and Pain Medicine, Tan Tock Seng Hospital (TTSH), National Healthcare Group, Singapore ${ }^{2}$ Department of Neuroanaesthesiology and Critical Care, All India Institute of Medical Sciences (AlIMS), New Delhi, India

J Neuroanaesthesiol Crit Care 2018;5:39-41.

The fifth biennial Congress of the Asian Society for Neuroanaesthesia and Critical Care (ASNACC) was held in Singapore, in conjunction with the 20th Annual General Scientific Meeting (GSM) of the Singapore Society of Anesthesiologists (SSA) during August 17 to 19, 2017, which was held at the Grand Copthorne Waterfront Hotel, located adjacent to the historic Singapore River. The biennial ASNACC Congress, Asia's leading conference on neuroanesthesia and neurocritical care, provides a common platform for thought leaders across diverse Asian countries to collaborate on improving care for the neurosurgical and neurological patient population. The Congress has previously been held in Beijing, China (2009); New Delhi, India (2011); Bali, Indonesia (2013); and Busan, South Korea (2015). This year, the fifth Congress edition took place in dynamic and progressive, compact yet cosmopolitan Singapore and featured eminent speakers from across Asia, as well as other distinguished international speakers including Prof. Andrew Kofke (University of Pennsylvania, Philadelphia, Pennsylvania, United States) and Prof. Adrian Gelb (University of California, San Francisco, California, United States). Leveraging on the apt theme "A Meeting of Minds," the scientific program consisted of a series of insightful plenary sessions, lectures, and panel discussions as well as a precongress neuromonitoring workshop to explore the challenging field of neuroanesthesia and neurocritical care.

The precongress "Multi-modality Intra-operative Neuromonitoring" workshop was attended by 36 local and overseas participants at the Tan Tock Seng Hospital, National Healthcare Group (Singapore). In this era of modern neuroanesthesia, there has been an ever-increasing use and recognition of the importance of neuromonitoring perioperatively. Up-to-date foundational knowledge on specific neuromonitoring modalities (transcranial Doppler, evoked potentials, multichannel bispectral index monitoring, and cerebral oximetry based on near-infrared spectroscopy) and, more importantly, their relevance to the role of the anesthesiologist in the operating theater and neurocritical care unit were covered in the workshop. Review lectures, supplemented by interactive case discussions as well as hands-on skill stations, were conducted
Address for correspondence Chong C. Ted, MBBS, MMed, FANZCA, Department of Anaesthesiology, Intensive Care and Pain Medicine, Tan Tock Seng Hospital (TTSH), National Healthcare Group, Singapore (e-mail: chin_ted_chong@ttsh.com.sg).

by Drs. Chong Chin Ted, Beatrice Lim, Mandy Lim, Chong Shang Yee, and Chen Xinying from Singapore and Prof. Girija Prasad Rath from India.

A record number of 378 delegates (349 registered participants and 29 invited faculty for the ASNACC program) from 15 countries and territories participated in the main Congress, and the geographic distributions of delegates are as follows: Singapore (155 delegates), Indonesia (62), South Korea (41), Philippines (31), India (27), Malaysia (14), Japan (11), Thailand (11), Australia (8), the United States (5), Myanmar (5), China/Hong Kong SAR/Taiwan (2/3/1), and Italy (2). Before the scientific program began in earnest, a brief opening ceremony was conducted during which Prof. See Jee Jian, organizing chairman of ASNACC and Prof. Kwek Tong Kiat, President, SSA, welcomed the participants. Prof. Tatang Bisri (Indonesia), president of ASNACC in his official opening speech outlined the growth of the wider neuroanesthesia scientific community across Asia and the importance of ASNACC as a bridge in building professional ties among member countries.

The first plenary lecture of day 1 was delivered by Prof. Adrian Gelb (United States) who challenged prevailing conventional thinking on cerebrovascular and intracranial pressure physiology. Entitled “Overturning Doctrines-Revisiting the Pressure Autoregulation Curve and Monroe-Kellie Doctrine," Prof. Gelb's lecture focused on two commonly held concepts in clinical practice (cerebral autoregulation and the intracranial pressure-volume curve) and reflected on some misrepresentations in the literature, especially textbooks, which has created a somewhat inaccurate mythology. Prof. Gelb urged the searching out of original primary sources of data and the avoidance of overreliance on predigested and interpreted information in book chapters and reviews. Following on, in the session dedicated to Neurocritical CareConsensus and Controversies, Prof. Andrew Kofke (United States) expounded on why "Functional Genonmics and Biomarkers" have generally been disappointing in the field of neuroprotection research. As many time-dependent pathophysiologic processes are involved in the final outcome after cerebral insult, the critical problem identified is the
Dol https://doi.org/ $10.1055 / \mathrm{s}-0037-1618327$ ISSN 2348-0548.
Copyright $\odot 2018$ Indian Society of Neuroanaesthesiology and Critical Care
License terms

(®) $\Theta \circledast$ 
application of therapy that is oriented to only single or few molecules or pathways of unknown comparative importance and with no due consideration of temporal changes after an ischemic insult. Subsequently he presented the findings of his proof-of-concept investigation to establish that, after cardiopulmonary bypass in humans, using functional genomics, multiple variably weighted proteins such as $\mathrm{S} 100 \beta$ and neurofilament $\mathrm{H}$ in association with single nucleotide polymorphisms are involved in the pathogenesis of perioperative human ischemic brain damage. Prof. Kofke then delivered his next topic on "Anaesthesia for Acute Ischemic Stroke: From Controversy to Consensus," which is a reference to the published SNACC Expert Consensus Statement on Anesthetic Management of Endovascular Treatment of Acute Ischemic Stroke. Prof. Kwek Tong Kiat (Singapore) completed the session with a lecture on "Brain Death Certification in Asia-Room for greater Standardisation?" This session was moderated by Dr. Vanitha Sivanaser (Malaysia).

The next session focused on various aspects on the specialized area of Awake Craniotomy. Highlighting the need for interprofessional collaboration from the perspective of a neurosurgeon, in his presentation "Awake craniotomy: Partnership between Neurosurgeon and Neuroanesthesiologist," Prof. Ng Wai Hoe (Singapore) spoke on the multiple problems and challenges unique to awake craniotomy that arise in the intraoperative phase of surgery. Prof. Dilip Kulkarni (India) explored "Awake Craniotomy-Pharmacological Options and Airway Strategies" whereas Dr. Sri Rahardjo (Indonesia) described "Scalp Blocks for Awake Craniotomy and Management of Craniotomy Pain." Prof. Tatang Bisri (Indonesia) championed the notion that simplicity is the ultimate sophistication in his discourse on "Techniques in Awake Craniotomy-Less Is More." His lecture advocated the use of mainly standard monitors of pulse oximetry, electrocardiogram, and blood pressure cuff, with invasive lines such as arterial lines and central venous catheters seldom indicated for straight-forward cases. Postoperative care can be safely conducted in the ward instead of the intensive care unit, with some larger centers even promoting day surgery. This session was chaired by Dr. Phuping Akavipat (Thailand). The last session of day 1 of the Congress, moderated by Dr. Kwon Jae-Young (South Korea), centered on "NeuroanaesthesiaImproving Outcomes." Prof. Adrian Gelb (United States) dissected the important short- and long-term aims in "Outcomes in Neuroanaesthesia-What Matters Most?" Much of the neuroanesthesia literature hitherto has focused on short-term perioperative outcomes such as extubation time, time to return to baseline neurologic assessment, intracranial pressure, brain relaxation, cerebral perfusion, and neurophysiologic monitoring. Although short-term outcomes are viable research targets and important in themselves, we must guard against assuming a relationship exists between these proximate measures and the long-term and arguably more clinically meaningful outcomes that patients actually desire, for example tumor recurrence, neurologic function or disability, quality of life, and survival. The call to action is for us to actively investigate what role, if any, anesthesia and anesthesiologists play in meaningful and enduring outcomes.
Indeed, anesthetic agents are often viewed in the context of being a double-edged sword, with both favorable and unfavorable effects. This is amply illustrated by the observation that beneficial "Neuroprotection and Ischaemic Preconditioning" (Prof. Mishiya Matsumoto, Japan) but also harmful "Neurodegeneration and Toxicity of Anaesthetics" (Dr. Putu Pramana, Indonesia) are both intrinsic characteristics of the anesthetic agents used. The field is an ever-evolving specialty and Dr. Chong Shang Yee (Singapore) spoke on "Latest Updates in Neuroanaesthesia" that complemented "Neuroanaesthesia for the Occasional Anaesthetist" by Dr. Phuping Akavipat (Thailand).

The plenary session entitled "Myths in Anesthetic depth monitoring" on the second day of the Congress was delivered by Prof. Matthew Chan (Hong Kong SAR) who also spoke on "Aneurysmal Subarachnoid Haemorrhage-Statins: Another Loss in Translation" in the following session on "Haemorrhagic Stroke." In common with many other interventions, statins represent another translational failure of presumed neuroprotective agents. Prof. Andrew Kofke (United States) discussed "Haemorrhagic Stroke-Implications for the Anaesthesiologist" both in the operating room and in the neurocritical care unit. Prognostic determinants such as intracranial hemorrhage expansion, blood pressure control, presence of anticoagulants, glycemic management, and surgical options were analyzed. Pertaining to anticoagulants in the setting of hemorrhagic stroke, Dr. Jensen $\mathrm{Ng}$ (Singapore) proposed several strategies for clotting normalization in "Warfarin, NOACs and Intracranial Haemorrhage-Strategies for Urgent Reversal." The monitoring for the effectiveness of reversal agents and outcomes that can be expected for this devastating neurological condition were also presented. This session was moderated by Dr. Geraldine Jose (Philippines). Postlunch topics delved mainly into the field of "Pituitary and Neurovascular." A comprehensive refresher talk by Dr. Chen Xinying (Singapore) on "Anaesthesia for Pituitary Surgery" was followed by "Pituitary Vasospasm and Non-SAH Vasospasm" by Prof. Hari Har Dash (India). In the interactive session chaired by Dr. Beatrice Lim (Singapore) on "Moyamoya Disease and Other Cerebrovascular Occlusive Disease" Prof. Kenji Yoshitani (Japan) and Prof. Hee-Pyoung Park (South Korea) shared their respective institution's experience and specific anesthetic considerations in managing pediatric and adult moyamoya patients undergoing direct or indirect cerebral revascularization/bypass procedures. In the final session of expert panel, Dr. Vanitha Sivanser (Malaysia), Dr. Geraldine Jose (Philippines), and Dr. Mandy Lim (Singapore) provided insightful expert opinion on "Difficult Airway Management in Neurosurgery" covering both the emergency setting of cervical spine injury and elective surgeries for craniofacial syndromes and acromegaly. The panel on "Postoperative Stroke" comprising Prof. Matthew Chan (Hong Kong SAR), Prof. Han Ruquan (China), and Dr. Chong Chin Ted (Singapore) laid out wide-ranging concerns such as incidence and predictors of postoperative stroke, diagnosis of overt versus covert stroke, prognosis and treatment of postoperative stroke, timing of surgery/anesthesia after stroke, and anesthetic depth and its relationship with neurologic 
outcomes such as stroke and delirium. "Neuro-prognostication and End-of-Life Issues" covering risk modeling from the IMPACT and the CRASH databases, ethical dilemmas specific to local cultural settings, and potential legal pitfalls were covered by Dr. Aaron Lee (Singapore), Prof. Thomas Lew (Singapore), and Prof. Hae-kyu Kim (South Korea).

The congress received a total of 79 scientific abstracts for poster presentations. The second prize for the Neuroanaesthesia and Neurocritical Care category was awarded to Malaysian delegate Dr. Mohamad Hasyizan Hassan of Hospital Universiti Sains Malaysia for the presentation on "Balanced
Fluid versus Saline-Based Fluid in Postoperative Severe Traumatic Brain Injury Patients: Acid-Base and Electrolytes Assessment." The first prize went to the South Korean submission by Dr. Young-kwon Ko of Chungnam National Hospital on "Multiple Sevoflurane Exposures during Pregnancy Do Not Affect Memory and Synaptic Plasticity in Offspring Mice."

The fifth Congress of ASNACC in Singapore concluded successfully with a preview of the fifth Congress in the city of Nara, by Prof. Kawaguchi Masahiko (Japan). In addition, there were thanks to all the invited speakers for their valuable contributions and to the delegates for their active participation. 